\title{
Biomechanical analyses of rat locomotion during walking and climbing as a base for the design and construction of climbing robots
}

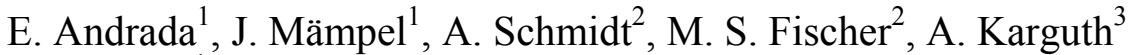 \\ \& H. Witte ${ }^{1}$ \\ ${ }^{I}$ Department of Biomechatronics, IMN MacroNano ${ }^{\circledR}$, \\ University of Technology Ilmenau, Germany \\ ${ }^{2}$ Institute of Systematic Zoology and Evolutionary Biology with Phyletic \\ Museum, Friedrich-Schiller-University Jena, Germany \\ ${ }^{3}$ TETRA Gesellschaft für Sensorik, Robotik und Automation mbH, \\ Ilmenau, Germany
}

\begin{abstract}
Ecological generalists, such as rats, may be used as biological paragons for the design and construction of adaptive light-weight climbing robots. We applied the inverse dynamic approach (IDA) on rats walking and climbing at different inclinations in order to estimate joint forces, torques and power in extremities and the trunk. If we understand the adaptive nature of small mammals' moving on sloped supports, via biomimetic transfer the principles uncovered may be applied to the design and construction of more adaptive climbing machines.
\end{abstract}

Keywords: biomechanics, rat locomotion, biologically inspired robots.

\section{Introduction}

The number of climbing robots has steadily increased during recent decades. Current systems that are able to climb have an overall mass ranging from $3 \mathrm{~kg}$ to $75 \mathrm{~kg}$. In comparison to their biological paragons, the locomotory capabilities of robots are quite constrained. The optimization of such robots requires a general understanding of the climbing process. This could be achieved by an examination of the biomechanical principles used by animals during horizontal, sloped and vertical locomotion. 
Following the argumentation in [1], the development of biomimetic robots should not only be based on morphological studies. Biomechanical and thus functional analyses should be done to achieve a model of climbing. On one hand, this leads to a basic understanding of locomotion. One the other hand, engineers get groundwork for their design processes. Furthermore, a verification of the model is possible by analyzing the climbing locomotion of robots. This process is shown in Figure 1. In the following the foci are on the biomechanical analyses and on the model of the climbing of rats. The common objective of the project "InspiRat" [3] is the development and construction of an autonomous climbing robot with a maximum mass of $1.5 \mathrm{~kg}$ based on the inspiration by biological paragons [4].

Evolution of mammalian locomotion is marked by many principles that match our claims. Among other things, spinal movements seem to play an important role during terrestrial locomotion and contribute up to $50 \%$ to the total propulsion [5], and the coordination between the body stem and extremities plays an important role for stable locomotion [6]. Rats, as ecological generalists (ubiquists), may be biological paragons for such adaptive light-weight climbers. In the present study we used the inverse dynamic approach (IDA) to estimate forces, torques and power in the extremities and trunk of rats walking and climbing at $60^{\circ}$ inclination. We have undertaken this study for several reasons. First, we seek to test whether gait modes change with inclination. Second, we want to test whether equivalent functional groups [7] (e.g. scapula-femur) in fore and hind limbs display similar torque patterns over the different locomotion tasks.

Third, we seek to bring more light about the coordination between the trunk and extremities in small mammals moving on sloped supports.

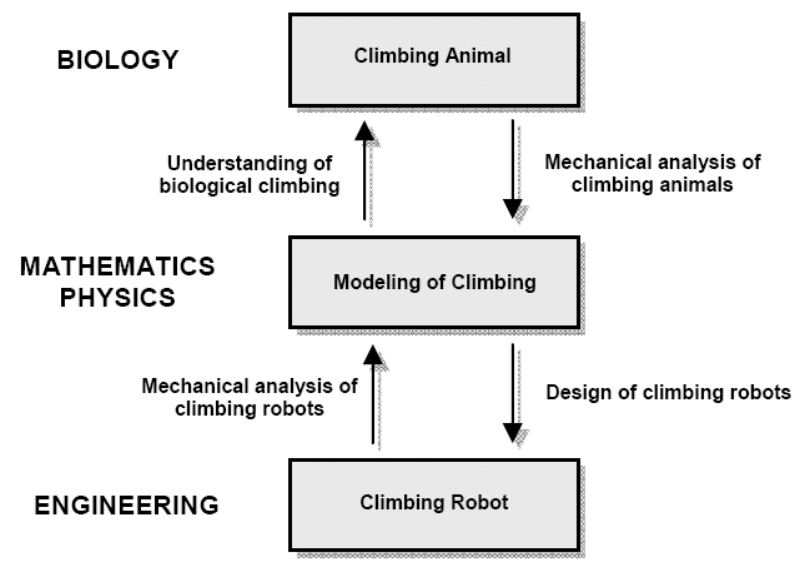

Figure 1: Design process in the development of a biologically inspired robot. The technical design process should be based on the model, deduced from biomechanical studies [2]. 
Finally, we expect to infer some biomechanical principles that allow us to offload a certain amount of computation for generating horizontal and sloped locomotion from the control system of our robot to its mechanical system.

\section{Materials and methods}

Animals: Metric, kinematic and kinetic data were obtained from two adult female rats (Rattus norvegicus) both weighing about $300 \mathrm{~g}$. The Committee for Animal Research of the State of Thuringia, Germany, approved animal care and experimental procedures. Animals were motivated to move across a simulated arboreal substrate at their preferred speeds. The cylindrical substrate was $2.00 \mathrm{~m}$ long and covered with cork, to allow claw penetration. It could be adjusted from horizontal to vertical orientation. Only trials in which the animals were travelling at steady speed were examined.

\section{Experimental setup}

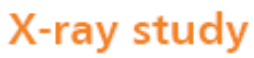

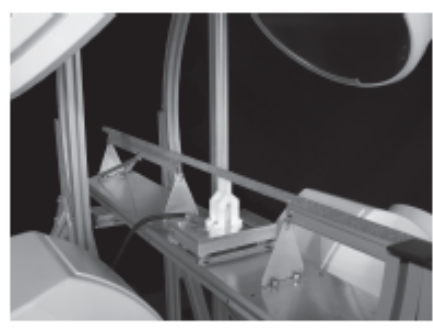

Mechanical link model

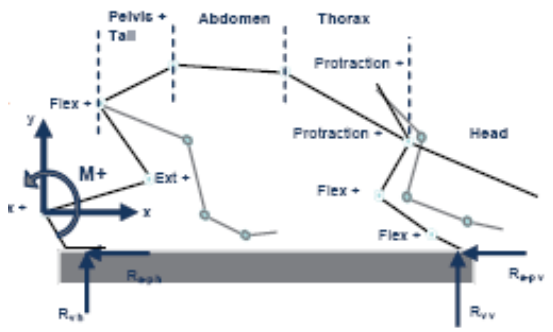

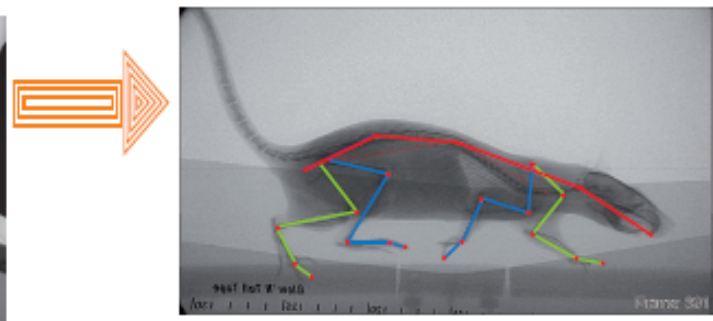

IDA

(Inverse Dynamic Approach)

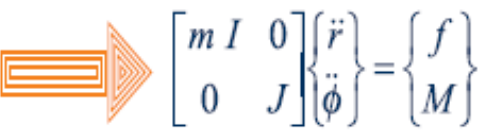

\footnotetext{
$m$ segment mass

$I$ identity matrix

$J$ inertial tensor about center of mass

$\vec{r} \quad$ linear acceleration

$\bar{\phi}$ angular acceleration

$f$ includes gravity and all distal and proximal forces

$M$ includes all torques due to forces and net muscular torques
}

Figure 2: Method to obtain torques and power in the extremities and trunk of rats walking and climbing at different inclinations. 
$X$-ray motion analysis: Kinematics and metrics were analyzed using biplanar X-ray imaging at Institute of Systematic Zoology and Evolutionary Biology with Phyletic Museum of the Jena University, Germany. The experimental setup consists of the biplanar X-ray system (Neurostar, Siemens ${ }^{\mathbb{B}}$ ) and two normal light high-speed cameras (SpeedCam Visario g2, Weinberger ${ }^{\mathbb{B}}$ ) (Figure 2: upper left). Both X-ray sources were arranged in a $45^{\circ}$ position, which allowed detailed perspectives of proximal skeletal elements such as the scapula. Cineradiographic images were taken at $1 \mathrm{kHz}$. The identification of joints was performed with software SimiMotion ${ }^{\circledR}$ (Figure 2: upper right).

Force data acquisition: Three-dimensional substrate reaction forces (SRF) were measured with a force-sensitive region integrated in the middle of the pole (55 mm length) (Fig.1 upper right). This force-transducer element was attached to the surface of one Kistler ${ }^{\circledR}$ force plate and separated from non-instrumented segments by $2 \mathrm{~mm}$ gaps. SRF's were collected at $500 \mathrm{~Hz}$. Analogue force data were amplified (8-Channel Charge Amplifier, Type 9865, Kistler ${ }^{\mathbb{B}}$ ), converted into a digital format via NI USB-6229 (National Instruments ${ }^{\mathbb{B}}$ ), and recorded with LABVIEW 8.2 (National Instruments ${ }^{\mathbb{B}}$ ). Force and X-ray analysis were synchronized electronically (post-trigger).

Mechanical link model: The mechanical model consists of 20 rigid segments, representing toes, feet, shank, thigh, hand, lower arm, upper arm, scapula, pelvis, abdomen, thorax and head. These segments were assumed to move only in the sagittal plane and to be linked via pin-joints. To calculate inertial properties (i.e. mass, position of centre of mass CoM, and inertial tensor about the centre of mass), the body stem of a rat cadaver (weight $300 \mathrm{~g}$ ) was dissected in four segments (inertia of the extremities was not taken in to account for calculations, since even in fast locomotion they only contribute maximally $15 \%$ to overall forces [7]).

The dissected segments (pelvis, abdomen, thorax and head) were measured and weighed; afterwards inertial properties were estimated matching each segment to geometrical forms. Extremities' morphometric data were obtained from [7].

IDA analysis: To enable synchronization of kinematical and force data and to reduce the noise in the twofold numerical derivation necessary for computing linear and angular accelerations, several techniques proposed in literature were applied (spline interpolation, mean and Butterworth filters). The trajectories of the segments' CoM were calculated from joint trajectories in combination with morphometric data. Absolute angle of each segment in space was defined counterclockwise from the substrate as positive. Joint angles were obtained from the scalar product of the vectors describing two adjacent segments. Internal forces of the joints and net torques about the CoM of limbs and body-stem segments were estimated applying the Newton-Eulerian approach (see Figure 2: bottom right). Torques are positive in counterclockwise direction. Joint power was estimated by multiplying the joint torque times the joint angular velocity. Positive power is defined as concentric work of a muscle group (torque and angular velocity have the same sign), negative power as eccentric. 
Following [7] the scapula was included in the analysis. The scapular fulcrum was modelled as an instantaneous centre of rotation (ICR), and therefore the net muscular torque was computed about it.

One of the challenges by computing torques on the trunk was whether shoulder, scapular or both torques should be transmitted to the thoracic segment. We decided to compare the result of the net torques in the thoracic-abdominal joint (T13) computed from propagation of forces and torques coming from foreand hindlimbs. Theoretically they should be equal but of an opposite sign. Practically they are usually different (personal communication N. Ogihara). Torques in the T13 joint computed using the sum of shoulder and scapular torques were found to be in-between 1.7 and 2.3 times larger than those obtained in the same joint computed from the hindlimbs. On the other hand by using torques generated in scapular or shoulder joint, differences were below $30 \%$. Therefore we decided to use the scapular torque, which is normally the bigger one [7].
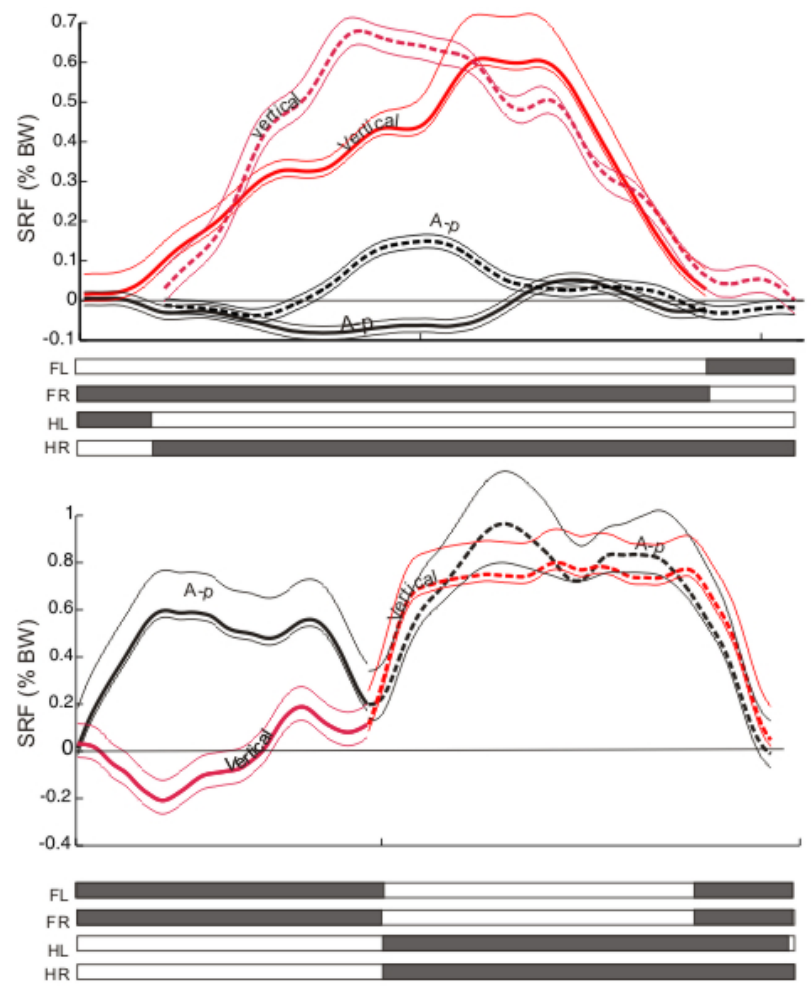

Figure 3: $\quad$ Substrate reaction force components in vertical (red lines (colour online only)) and anterior-posterior (black lines) directions. Displayed is one representative trial and the maxima and minima values observed for four trials. Top: horizontal locomotion, bottom: sloped $60^{\circ}$. Forelimbs (solid line), hindlimbs (dashed line). 
During in-phase gaits (both fore or both hind limbs touch the ground simultaneously) torques and power were calculated using the total SRF and the kinematics of the left extremities. In order to obtain the torques and power acting in each joint the results were be divided by 2 . Signal processing, the model and the IDA were implemented in Matlab ${ }^{\mathbb{R}}$.

\section{Results}

The rats prefer symmetrical gaits (walk, trot) during locomotion on horizontal and substrates with up to $30^{\circ}$ inclination. Equivalent functional groups in foreand hindlimbs displayed similar torques and power patterns during horizontal walking. They switched to synchronous gaits (gallop) above $30^{\circ}$ inclination In Figure 3 the substrate reaction forces are shown for locomotion at horizontal (top) and $60^{\circ}$ inclination (bottom). At horizontal locomotion the forces in a-p direction are low. The values of the forces do not differ between front and hind limbs. The value of the vertical component is about $0.7 \mathrm{BW}$. Hence, a dynamic behaviour could be observed.

At $60^{\circ}$ inclination, forces are ruled by gravity. For the forces orthogonal to the substrate, the component provoked by gravity is $0.5 \mathrm{BW}$, for a-p-forces it is 0.86 . For both front limbs together, an a-p-force of 0.6 times BW was measured. Due to the fact, that this propulsion force is below the gravitational share, the rat decelerates in this phase. During the stance period of the hind limb, the propulsion force is about $0.9 \mathrm{BW}$ and compensates gravitational force.

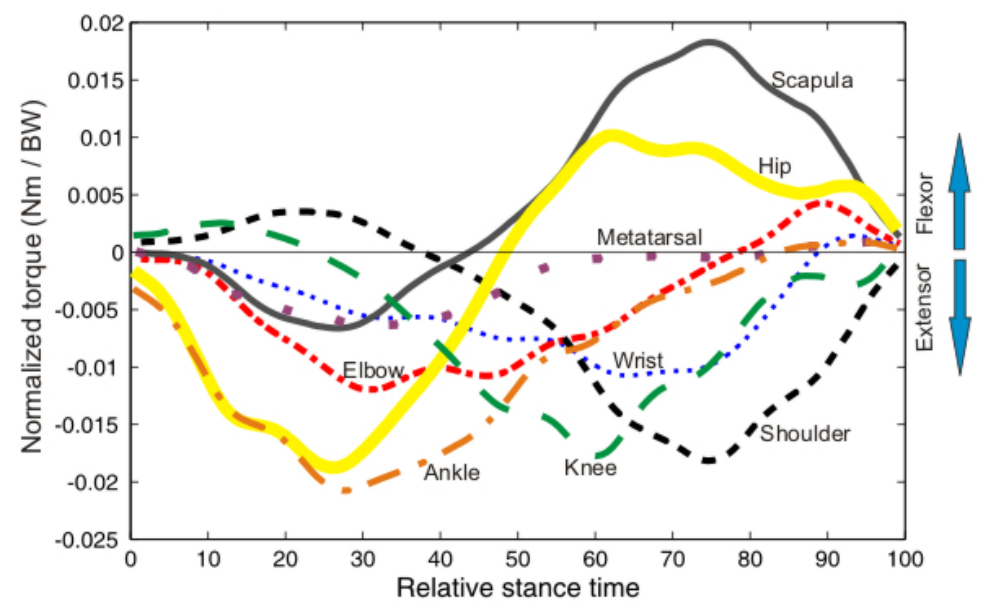

Figure 4: Normalized limb torques estimated during horizontal locomotion of a rat. Displayed is one representative trial. The variability observed for four trials was about $15 \%$ of the maximal values. Data correspond to the results of preliminary studies [7]. 

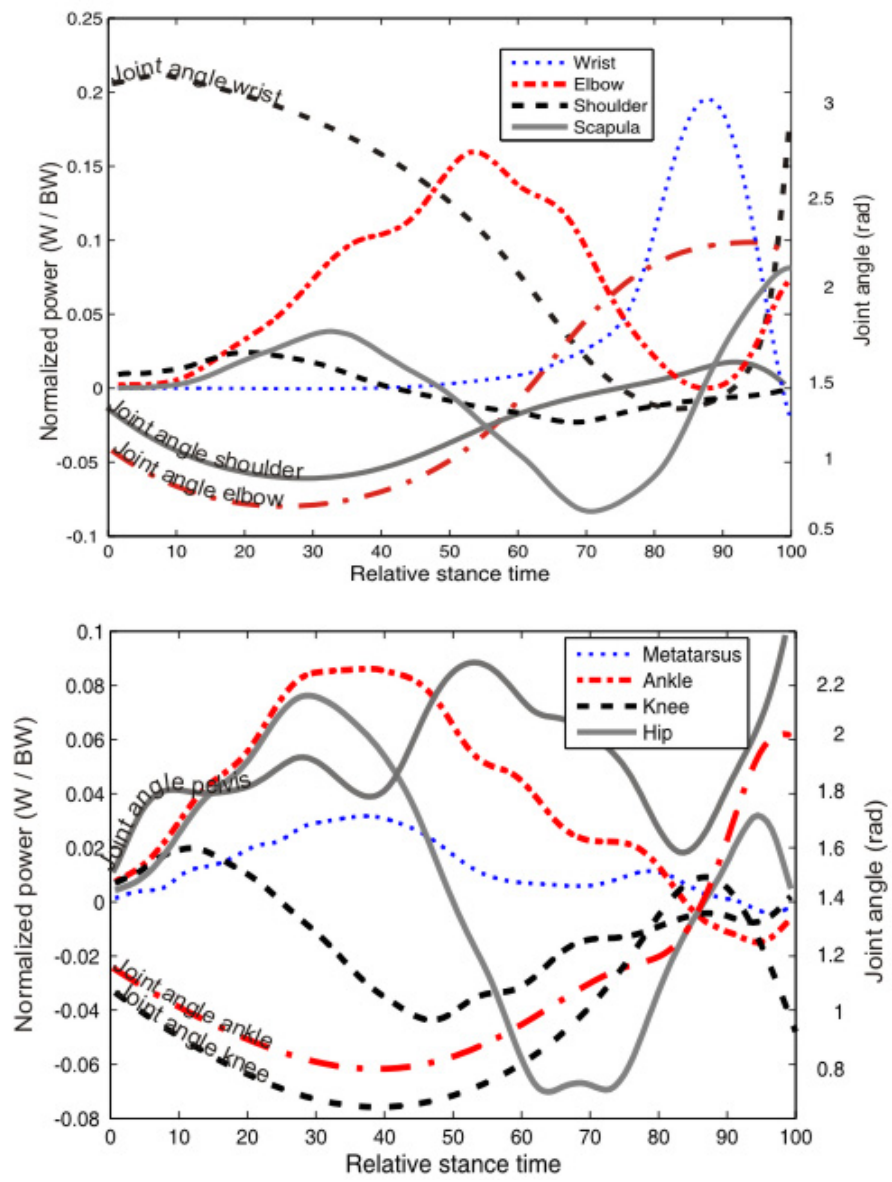

Figure 5: Normalized limb joint powers and joint angles estimated during horizontal locomotion of a rat. Displayed is one representative trial. The variability observed for four trials was about $15 \%$ of the maximal values for the proximal segments and about $30 \%$ for the distal segments.

The time course of torques becomes different during locomotion on substrates with $60^{\circ}$ inclination. During horizontal locomotion hip and scapula exhibit a biphasic torque path while hip extensors and scapular retractors (e.g. M. levator scapulae ventralis, M. acromiotrapezius) worked until $50 \%$ of the stance phase (st-ph) concentrically (Figure 4:). During the second part of the st-ph hip flexors and scapular protractors (e.g. M. spinotrapezius) worked eccentrically. Torques and power patterns for knee and shoulder joints revealed an asymmetrical biphasic behaviour; shank and upper arm were concentrically flexed, respectively, retracted until approximately $30 \%$ of the st-ph, then eccentrically 

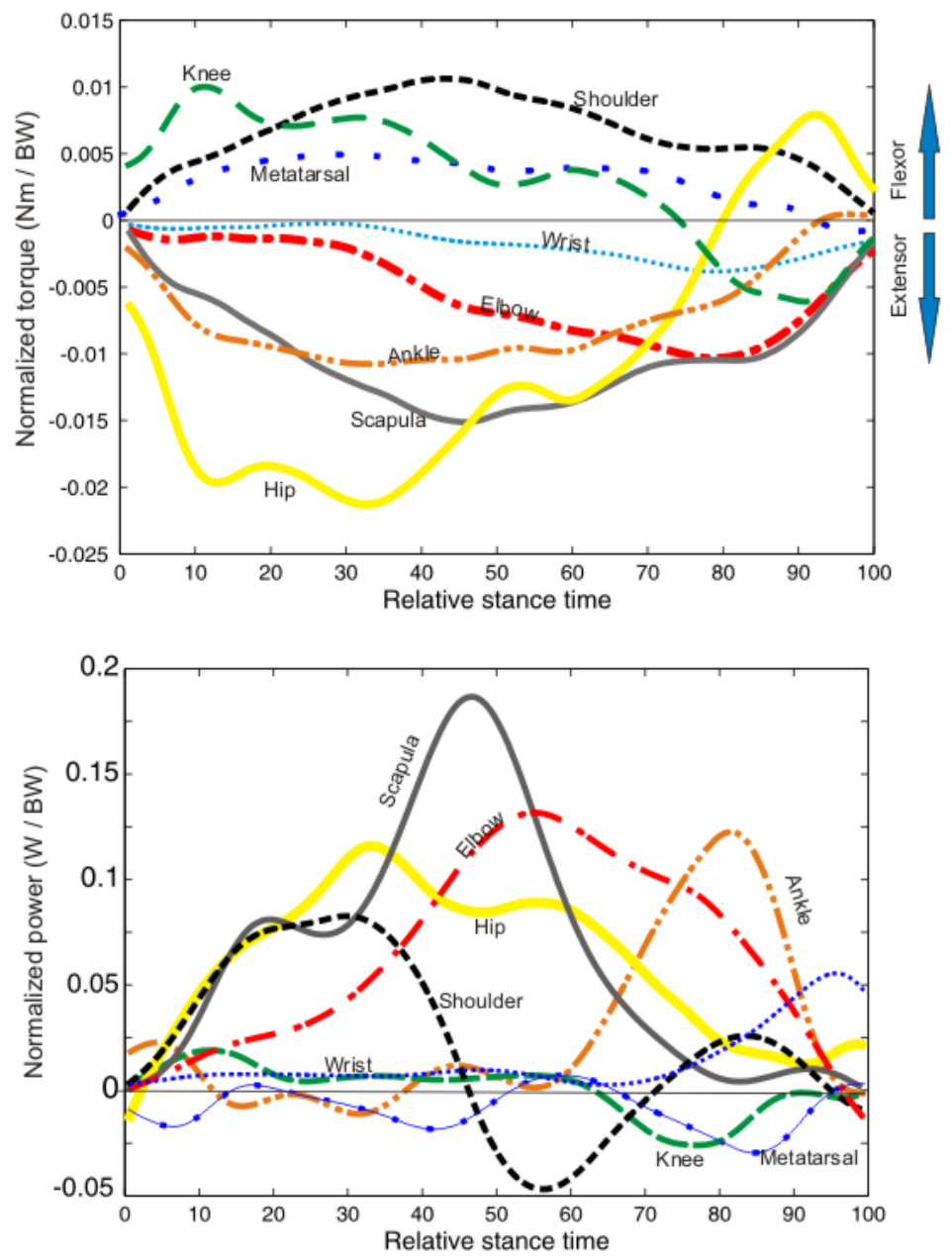

Figure 6: Normalized limb joint torques and powers estimated during the sloped locomotion of a rat $\left(60^{\circ}\right)$. Displayed is one representative trial. The variability observed for four trials was about $15 \%$ of the maximal values for the proximal segments and about $20 \%$ for the distal segments.

extended and protracted, respectively. On the other hand torques and power curves of ankle and elbow joints were monophasic (plantar flexors and lower arm extensors worked concentrically). Only for the most distal joints torques and power patterns were found to be different (metatarsophalangeal and wrist joints).

If inclination increases, joint torques and power paths in the forelimbs become monophasic (half sinus), and the joint power remains positive during the entire st-ph. More in detail, the changes between concentric and eccentric work 

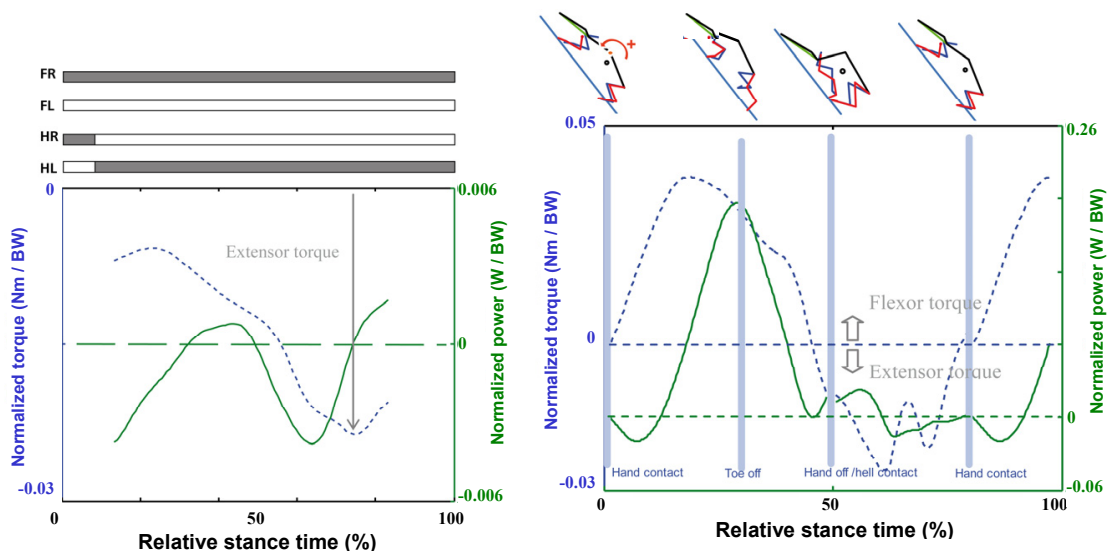

Figure 7: $\quad$ Normalized limb joint torques (dashed line) and powers (solid line) estimated during horizontal (left) and $60^{\circ}$-sloped (right) locomotion of a rat. Displayed is one representative trial.

in the most proximal muscle groups were shifted from approximately $50 \%$ of the st-ph during horizontal locomotion to almost $100 \%$ during inclined locomotion $\left(60^{\circ}\right)$.

In Figure 6 normalized joint torques and powers in fore- and hindlimbs during climbing at $60^{\circ}$ inclination are presented. As mentioned above, torque patterns observed in the knee and the hip differ in relation to their equivalent functional group; they remain biphasic (Fig. 5).

By comparing torque patterns obtained during horizontal locomotion and $60^{\circ}$ inclination, two important findings can be addressed: 1 . As inclination increases, torque maxima in the proximal pivot (scapula) become larger than those of the shoulder joint. 2. The maximum joint torques generated during climbing $\left(60^{\circ}\right)$ did not differ from those obtained during horizontal locomotion. By contrast the positive power exerted by the joints increases significantly.

For the first time, trunk torque and power patterns are described for rats while walking or climbing. In the first $50 \%$ of the st-ph during horizontal locomotion, the trunk is extended whereas the torque in the thoracic-abdominal joint stays nearly zero (Figure 7 left). It seems that the forces and torques acting on the proximal joints of the fore- and hindlimbs achieve this extension, whereas the forelimbs seem to act as a spring-mass-element. As a result the CoM descends. This strategy may contribute to a stable locomotion.

In the second half of the st-ph the trunk is flexed, while in T13 an extensor torque, with a negative power, acts simultaneously. This suggests that dorsal musculature works eccentric in order to control trunk flexion. With increasing inclination, the concentric work of the abdominal musculature becomes crucial. During climbing $\left(60^{\circ}\right)$ forelimbs push downwards parallel to the substrate and a flexor torque appears at the T13 joint (Figure 7 right). Rapidly the power becomes positive, indicating that the abdominal musculature works concentric. 
Interestingly, at moment the flexor torque has reached the maximal value, power changes from negative to positive.

Subsequently the maximum positive power coincides with lift off, whereas the abdomen is shifted upwards. With the beginning of the support phase of the hind limbs thorax and pelvis are extended, forelimbs are protracted, while SRFs are directed to the CoM. The SRFs transmitted in the hind limbs become propulsive at touch down of the forelimbs. During the swing phase of the forelimbs torques in T13 remain negative (extensor), but the power oscillates between positive and negative, indicating that dorsal musculature may control the trunk extension during this phase.

\section{Discussion}

The present investigation can be understood as a logical continuation of previously published studies on locomotion of small therian mammals on flat ground [5-7]. This study presents for the first time torques and power paths of rats during arboreal locomotion.

Rats display two different locomotion strategies in dependence of the slope angle. During locomotion on horizontal substrates as well as at moderate inclinations $\left(30^{\circ}\right)$ rats prefer symmetrical gaits. With increasing inclination they switch to synchronous gait (gallop).

During horizontal locomotion both extremities contribute almost in the same way to body propulsion. As observed previously in other mammals forelimbs generate larger braking forces [9]. Furthermore, this study shows that during horizontal locomotion torques and power for the most proximal joints in forelimbs are very akin to those displayed by the hind limbs (max. value and shape). During horizontal locomotion forelimbs seem to be coordinated to yield a spring-like behaviour. Our observations agree with [10], showing that the most distal joints (ankle and wrist) seem to be the most important contributors for the spring-like behaviour, providing ankle extensors and their long tendons an obvious site for the storage and release of mechanical energy. On the other hand the most proximal joints seem to contribute more to the actuation, these differences becoming even more obvious as inclination increases. Surprisingly, maximal joint torques during walking and climbing did not differ significantly.

If one represents the complete st-ph of the rat by means of the motions of its $\mathrm{CoM}$, the whole behaviour could be also represented as a spring-mass system [11]. Our results show, however, that this behaviour is strongly supported also due to a possibly "passive" trunk extension in the early st-ph (in agreement with [12] we expect no dorsal muscular activation during the first half of the st-ph), provoking that the CoM becomes descend, and followed by a controlled trunk flexion in the second half of the st-ph. This flexion was also associated with the forward displacement of the CoM, as a way to adjust the angle of attack of the forelimbs and thus to use mechanical self-stabilization mechanisms [6].

During arboreal locomotion on the inclined substrate the rat's locomotion becomes markedly "hindlimb dominated" as observed in primate quadrupedal locomotion (cf. [13]). In observations, an inclination between $30^{\circ}$ and $60^{\circ}$ marks 
the boundaries of the symmetrical gaits. We propose that at this point rats reach the maximal joint torques they can exert, especially in the hind limbs. Thus in order to climb more sloped substrates, as observed at $60^{\circ}$, they may have to switch to the more quasistatic in-phase gait, which permits them to exert more propulsional force using similar joint torques. Furthermore, our findings show that at those inclinations the active co-work of the extremities with the trunk becomes crucial. From our results it is clear that during forelimb st-ph the concentric work of the abdominal musculature (presumably through the contraction of the $M$. rectus abdominis) contributes to the propulsion, while dorsal muscles are used during the hind limb st-ph as springs and control-like elements. This behaviour seems to be an indication for a change from a walking to a climbing gait. Rat switches, maybe, due to maximum muscle forces from a dynamic walk to a quasistatic behaviour during climbing.

\section{Transfers into a concept for climbing robots}

The rat has a complex kinematical structure. Each leg consists of four segments. The spine is highly flexible, also consisting of many parts.

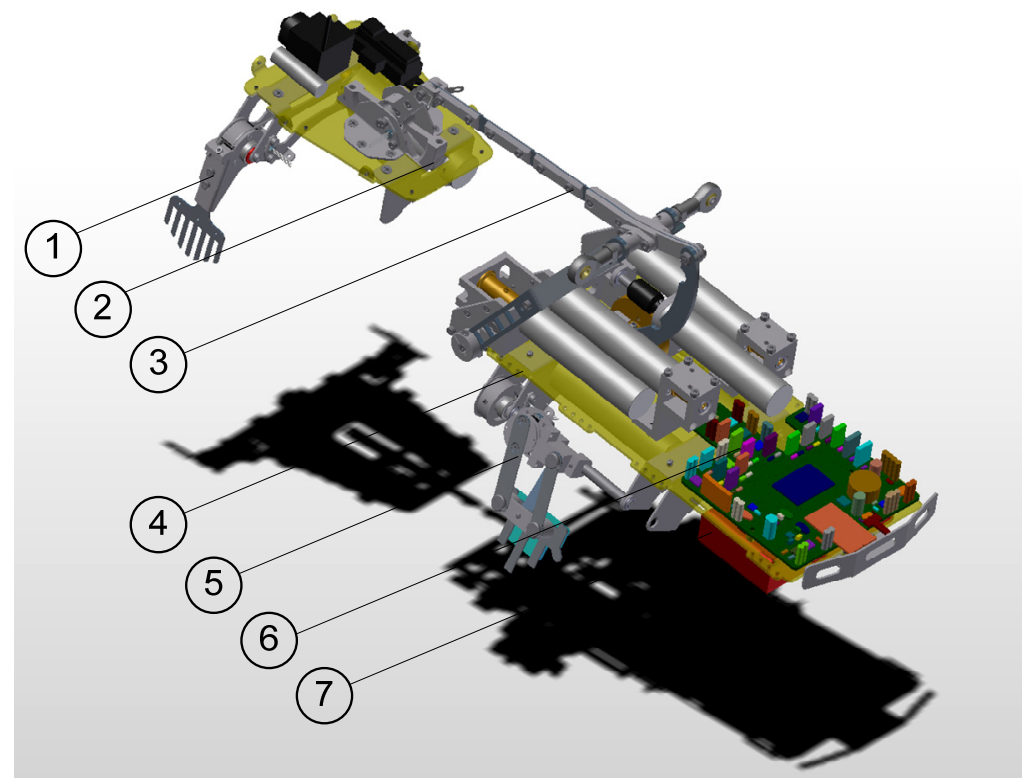

Figure 8: CAD-drawing of "RatNic" robot - the systems basically consists of a smaller front body (2) with gripper (1) and a bigger hind body (4) with gripper (5), supply unit (7) and electronics (6). These bodies are connected by a flexible spine (3). The actuators are placed in the hind body and the forces are transmitted via Bowden-cables (not in the figure). 
The degrees of freedom (DOF) of our rat's model are 20. The motion of a rat is quite complex. Hence, a transfer by copying the kinematical structure to a robot is extensive. In our opinion, the overall degree of freedom has to be reduced for an understanding of climbing process and for design of a robot.

Our climbing robot at least is designed with a DOF of 4 plus two additional DOF for the gripper. The two hind and two fore limbs are reduced in each case to a gripper. The relative motion between the two grippers is achieved by an actuated linkage mechanism. Hence, the kinematical structure is reduced. The frontal body carries the actuator for the front gripper; the hind body carries the actuators for the spine as well as all necessary electronic components. Due to the observed hind limb dominance during climbing at $60^{\circ}$ inclination, mass is concentrated in the hind body. The relative distribution is about $30 \%$ in the front and $70 \%$ in the hind body.

It has been shown, that the propulsion forces are mainly generated in the proximal joints and in the trunk. Generation of locomotion is mainly done by the trunk. Furthermore, it could be observed that the trunk of the rat acts like a spring. Thus in the robot elastic elements are integrated. If the light front body is moved upwards, energy is stored in the spring. This energy could be released during the movement of the hind body. By this means, a support for the actuator is achieved.

\section{Conclusion}

In our biomechanical analyses it could be observed, that rats change from walking to climbing. The range of this changeover occurs between $30^{\circ}$ and $45^{\circ}$ inclination. At $60^{\circ}$ inclination the locomotion becomes dominated by hind limbs, due to the necessity of dealing with gravitational forces. In addition the generation of motion was observed to be mainly caused by the trunk and by the proximal muscles of the limbs.

Based on our findings, we finally suggest the following points for the design process of a climbing robot:

1. A quasi-static motion is adequate for climbing at high inclinations.

2. The complex 3D-motion can be reduced to a planar motion.

3. Due to a reduction of the degrees of freedom, the forelimbs as well as the hind limbs could be combined.

4. The motion can be generated in the centre of robot and does not need to be mainly produced in extremity-like parts.

5. Elastic elements should be integrated in the trunk to store energy and to support the actuators during climbing.

\section{Acknowledgements}

This work is supported by grant 01RI0633 of the German Federal Ministry of Education and Research and German Aerospace Center. We thank Danja Voges for the support in editing the graphics. 


\section{References}

[1] H. Witte, H. Hoffmann, R. Hackert, C. Schilling, M.S. Fischer, H. Preuschoft, (2004), "Biomimetic robotics should be based on functional morphology". J Anat, 204(5), 331-342.

[2] Mämpel, J., Eisold, R., Kempf, W., Schilling, C. \& Witte, H. "A Modular Concept for a Biologically Inspired Robot". Robot Motion and Control 2009, pp. 391-299, 2009

[3] Inspirat project, "http://www.inspirat.de".

[4] J. Mämpel, E. Andrada, H. Witte, C. Trommer, A. Karguth, M.S. Fischer; D. Voigt; S. Gorb, , "Inspirat - Towards a biologically inspired climbing robot for the inspection of linear structures". In: Advances in Mobile Robotics, pp. 206 - 213, 2008.

[5] M.S. Fischer, and R. Lehmann, "Application of cineradiography for the metric and kinematic study of in-phase gaits during locomotion of the pika”. Zoology Vol. 101, pp. 12-37, 1998.

[6] R. Hackert, N. Schilling, M.S. Fischer, "Mechanical self-stabilization, a working hypothesis for the study of evolution of body proportions in terrestrial mammals?", C.R. Paleovol, Vol. 5, pp. 541-549, 2006.

[7] H. Witte, J. Biltzinger, R. Hackert, N. Schilling, M. Schmidt, C. Reich, M.S. Fischer, "Torque patterns of the limbs of small therian mammals during locomotion on flat ground", J Exp Biol, Vol. 205, pp. 1339-1353, 2002.

[8] D.A. Winter, Biomechanics and Motor Control of Human Movement, John Wiley \& Sons, New York, 1990.

[9] B. Demes, S.G. Larson, J.T. Stern Jr., W.L. Jungers, A.R. Biknevicius, D. Schmitt, "The kinematics of primate quadrupadalism: hindlimb drive reconsidered", J Hum Evol, Vol. 26, pp. 353-374, 1994.

[10] D.V. Lee, M.P. McGuigan, E.H. Yoo, A.A: Biewener, "Compliance, actuation, and work characteristics of the goat foreleg and hindleg during level, uphill, and downhill running", J Appl Physiol, Vol. 104, pp. 130-141, 2008.

[11] R. Blickhan, "The spring-mass model for running and hopping", $J$ Biomech, Vol. 22, pp. 1217-1227, 1989.

[12] D.A. Ritter, P.N. Nassar, M. Fife, D.R. Carrier, "Epaxial muscle function in trotting dogs". J Exp Biol, Vol. 204, pp. 3053-3064, 2001.

[13] E. Hirasaki, H. Kumakura, S. Matano, "Biomechanical analysis of vertical climbing in the spider monkey and the Japanese macaque", Am J Phys Anthropol, Vol. 113, pp. 455-472, 2000. 